\title{
Modelagem e simulação de elutriadores em série utilizando o Scilab
}

\author{
Modeling and simulation of serial elutriators using Scilab \\ Modelado y simulación de elutriadores en serie utilizando Scilab
}

Recebido: 09/08/2021 | Revisado: 14/08/2021 | Aceito: 16/08/2021 | Publicado: 18/08/2021

\author{
Amanda Branco e Silva \\ ORCID: https://orcid.org/0000-0002-1697-3136 \\ Universidade Federal do Triângulo Mineiro, Brasil \\ E-mail: amandabrancoes@gmail.com \\ Ana Paula Brandão \\ ORCID: https://orcid.org/0000-0002-1021-450X \\ Universidade Federal do Triângulo Mineiro, Brasil \\ E-mail: anapbrandao@live.com \\ Flávia Karlayne de Melo \\ ORCID: https://orcid.org/0000-0001-9212-0111 \\ Universidade Federal do Triângulo Mineiro, Brasil \\ E-mail: flaviakarlayne@ hotmail.com \\ Hudson Henrique Alves da Silva \\ ORCID: https://orcid.org/0000-0002-3342-4729 \\ Universidade Federal do Triângulo Mineiro, Brasil \\ E-mail: hudson03@hotmail.com \\ Italo Andalecio Gontijo \\ ORCID: https://orcid.org/0000-0003-4684-3217 \\ Universidade Federal do Triângulo Mineiro, Brasil \\ E-mail: italoandalecio@gmail.com \\ Leonardo Paganelli Meneghelli \\ ORCID: https://orcid.org/0000-0002-1018-9199 \\ Universidade Federal do Triângulo Mineiro, Brasil \\ E-mail: leonardopmeneghelli@gmail.com \\ Matheus Henrique Batista Palmieri da Silva \\ ORCID: https://orcid.org/0000-0002-3740-3474 \\ Universidade Federal do Triângulo Mineiro, Brasil \\ E-mail: matheushbps@outlook.com \\ Pedro Henrique Silva Mauad \\ ORCID: https://orcid.org/0000-0002-9740-6909 \\ Universidade Federal do Triângulo Mineiro, Brasil \\ E-mail: phsmauad@hotmail.com \\ Davi Leonardo de Souza \\ ORCID: https://orcid.org/0000-0002-1995-9057 \\ Universidade Federal do Triângulo Mineiro, Brasil \\ E-mail: davi.souza@uftm.edu
}

\begin{abstract}
Resumo
Em Operações Unitárias de Engenharia Química, entre os inúmeros equipamentos que podem ser utilizados na etapa de separação de sólidos indesejados daqueles que contém valor agregado, estão os elutriadores. O funcionamento destes equipamentos é baseado na diferença de tamanho e/ou densidade das partículas alimentadas no sistema, as quais ficam retidas no equipamento ou são carregadas pelo fluido de arraste, dependendo das características físicas do sistema particulado. Os elutriadores são usualmente operados em série e são amplamente utilizados na indústria de mineração. O presente estudo visa analisar o comportamento de um sistema de três elutriadores em série, cujas partículas alimentadas se diferem no tamanho e na densidade, utilizando o Scilab como principal ferramenta de análise. Ao estudar o comportamento do sistema frente à variação dos parâmetros alfa $(\alpha)$, velocidade mínima de fluidização (Umf) e vazão do sistema (Q), foi analisada a influência das alterações nas variáveis de massa retida no leito (W(ri)) e constante de elutriação (k(ri)).
\end{abstract}

Palavras-chave: Arraste; Elutriação; Simulação.

\section{Abstract}

In Chemical Engineering Unit Operations, among the countless pieces of equipment that can be used in the stage of separation of unwanted solids, those that contain added value are in the elutriators. The operation of these equipment is based on the difference in size and/or density of the particles fed into the system, which are retained in any equipment or are carried by the drag fluid, depending on the physical characteristics. Elutriators are usually operated in series and are widely used in the mining industry. This study aims to analyze the behavior of three elutriators in series, separate 
the fed particles if they differ in size and density, using Scilab as the main tool. When studying the behavior of the system in face of the variation of the alpha $(\alpha)$, minimum fluidization velocity (Umf) and system flow (Q) parameters, the influence of changes in the variables of mass retained in the bed (W (ri)) and elutriation constant (k (ri)) were analyzed.

Keywords: Drag; Elutriation; Simulation.

\section{Resumen}

En Operaciones Unitarias de Ingeniería Química, entre los innumerables equipos que se pueden utilizar en el paso de separar los sólidos no deseados de los que contienen valor agregado, se encuentran los elutriadores. El funcionamiento de estos equipos se basa en la diferencia de tamaño y / o densidad de las partículas que ingresan al sistema, las cuales son retenidas en el equipo o transportadas por el fluido de arrastre, dependiendo de las características físicas del sistema particulado. Los elutriadores generalmente se operan en serie y se usan ampliamente en la industria minera. El presente estudio tiene como objetivo analizar el comportamiento de un sistema de tres elutriadores en serie, cuyas partículas alimentadas difieren en tamaño y densidad, utilizando Scilab como principal herramienta de análisis. Al estudiar el comportamiento del sistema frente a la variación de los parámetros alfa $(\alpha)$, velocidad mínima de fluidización (Umf) y flujo del sistema (Q), la influencia de los cambios en las variables de masa retenida en el lecho (W (ri)) se analizó y la constante de elutriación (k (ri)).

Palabras clave: Arrastrar; Elutriación; Simulación.

\section{Introdução}

A mineração no Brasil remonta aos tempos coloniais, quando o ouro era cobiçado e buscado no interior do país. O Brasil é reconhecido por ser um dos principais produtores de minérios do mundo, produzindo aproximadamente 70 substâncias minerais, segundo o DNPM. Os principais minérios explorados no país são o minério de ferro, ouro, nióbio e alumínio. No ano de 2015, segundo o DNPM, o Brasil foi o principal produtor de nióbio do mundo, destacando-se por representar cerca de 97,3\% da produção mundial.

No processo de mineração o solo é explorado e diversas operações são empregadas para a obtenção do minério de interesse, entre elas estão a britagem, peneiramento, moagem, sedimentação, elutriação, hidrociclonagem, filtração etc.

A atuação profissional de um Engenheiro Químico está intimamente ligada ao desenvolvimento de Operações Unitárias, as unidades interdependentes que compõe os mais diversos processos industriais. Dentro das operações fluidodinâmicas que envolvem sistemas particulados, destacam-se a filtração, a sedimentação, a fluidização, leitos (fixos, fluidizados ou de jorro) e a elutriação (Cremasco, 2015).

A elutriação consiste em operações de separação baseadas na diferença entre a velocidade média do fluido e a velocidade terminal da partícula (Cremasco, 2014). Sendo assim, as partículas com diferentes diâmetros e massas específicas apresentam distribuições de velocidades distintas, o que é utilizado como fator determinante na separação das frações de sólidos (Silva, 2016).

Um elutriador consiste em uma coluna de diâmetro conhecido, onde a amostra de partículas é alimentada perto do topo. O fluido (na maioria das vezes a água) é alimentado com uma vazão volumétrica (Q) perto da base da coluna (Cremasco, 2014).

Existem duas maneiras para que a elutriação ocorra. Se o material for homogêneo, a separação ocorrerá devido a diferença de diâmetro entre as partículas; já se a mistura for heterogênea, a separação se dará pelas diferenças da velocidade terminal das partículas e das densidades (Foust, et al., 2011).

É possível que a elutriação aconteça com a utilização de um fluido gasoso (ar, na maioria das vezes) ao invés de líquido, técnica amplamente utilizada para determinar o tamanho de partículas de poeiras contaminantes. A grande vantagem deste tipo de elutriação é efetuar a medida instantânea das partículas separadas (Gomide, 1980).

Para entender o funcionamento de uma operação de elutriação é necessário avaliar os regimes fluidodinâmicos durante a fluidização, que dependem das características físicas da fase particulada (distribuição granulométrica, tamanho médio das partículas, forma e massa específica) e da fase fluida (viscosidade dinâmica e massa específica), assim como das condições operacionais da coluna (temperatura e vazão do fluido, compactação da fase particulada, altura e diâmetro) (Klinger, et al., 2017). 
Os regimes podem ser identificados como fluidização homogênea, fluidização borbulhante, fluidização do tipo slug, fluidização turbulenta e fluidização rápida (Cremasco, 2014). O autor reitera que a partir da fluidização turbulenta, um pequeno acréscimo na velocidade superficial do fluido acarreta na elutriação, ou seja, o arraste da fase particulada. A Figura 1 ilustra os regimes de fluidização.

Figura 1. Ilustração de regimes de fluidização.

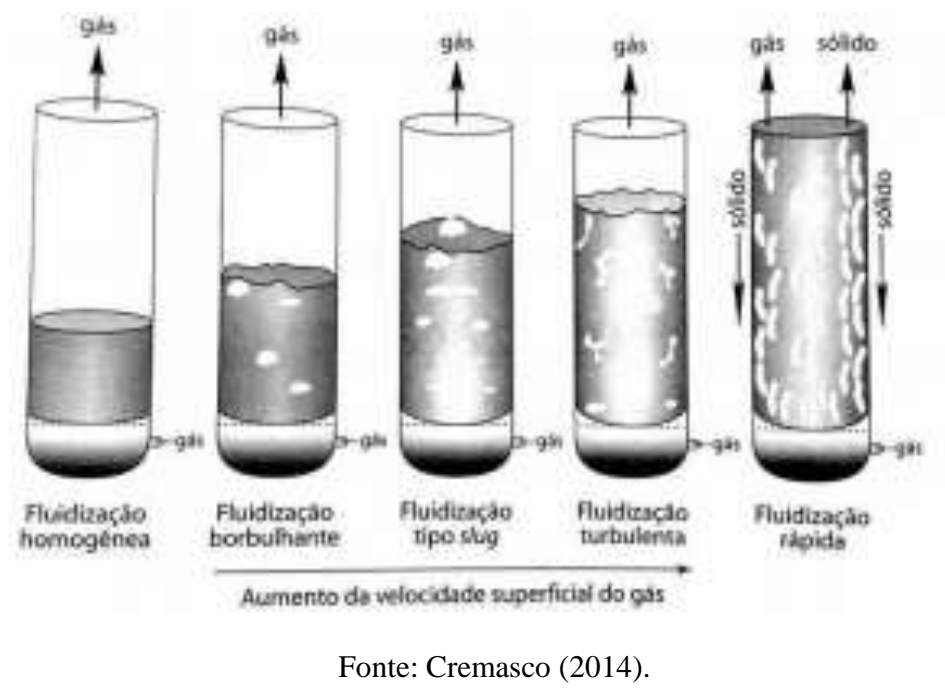

É importante destacar que os elutriadores são comumente utilizados na separação de rejeitos minerais. Estudos mostraram que é possível realizar a separação entre pirita (FeS2) e carvão (e outros minerais) por elutriação com água, dada a diferença de densidade entre os particulados (Englert \& Rubio, 2011). Essa operação é de extrema importância, visto que minimiza as chances de acontecerem sérios danos ambientais após o descarte dos rejeitos do beneficiamento de carvão mineral, como a drenagem ácida de minas.

Keleoneker, 2011, que também objetivou em suas pesquisas separar a pirita (FeS2) do carvão por elutriação, salientou que a pirita é a principal matéria prima para a elaboração de reagentes e enxofre, dado os teores suficientemente elevados do componente no rejeito mineral. Sendo assim, propôs a reutilização econômica do rejeito piritoso para gerar produtos comercializáveis, além de minimizar o impacto ambiental.

Silva, 2016, estudou e comprovou a possibilidade de separação de uma mistura composta por água e frações de argila, silte e areia por elutriação, após o descarte de sedimentos retirados de processos de dragagem no Porto do Rio Grande, de dezembro de 2013 a janeiro de 2014. O autor ainda analisou por métodos computacionais (fluidodinâmica computacional - CFD) os parâmetros operacionais do equipamento, objetivando uma alta eficiência de separação.

Um estudo relativo à simulação da separação de dióxido de titânio (TiO2) foi feito com a intenção da avaliação da aplicabilidade da elutriação por meio de sistemas gasosos (Trabzuni, 2009), base da modelagem dos elutriadores utilizada no presente caso.

\section{Metodologia}

Inicialmente, foi projetada uma série de três elutriadores, cada qual com $50 \mathrm{~cm}$ de altura, cujo objetivo era separar uma mistura de três partículas de densidades distintas. A separação das partículas em cada elutriador seguiu a ordem de densidade: no primeiro elutriador, ficaram retidas as partículas mais densas (7,4 g. m-3); no segundo, as partículas de densidade intermediária (5,5 $\mathrm{g} \mathrm{cm}-3)$, e no terceiro, por fim, as partículas menos densas $(2,2 \mathrm{~g} \mathrm{~cm}-3)$.

Os cálculos utilizados para o projeto dos diâmetros dos elutriadores são descritos a seguir. A equação 1 representa o 
cálculo do primeiro parâmetro da esfericidade (K1).

$K_{1}=0,843 \log _{10}\left(\frac{\varphi}{0,065}\right)$

$K_{1}=0,843 \log _{10}\left(\frac{\varphi}{0,065}\right) K_{1}=0,843 \log _{10}\left(\frac{\varphi}{0,065}\right)$

Em que $\varphi$ é a esfericidade. A equação 2 é referente ao cálculo do segundo parâmetro da esfericidade (K2).

$K_{2}=5,31-4,88 \varphi$

$K_{2}=5,31-4,88 \varphi K_{2}=5,31-4,88 \varphi$

A equação 3 descreve o cálculo do parâmetro do número de Reynolds $\left(\mathrm{CdRe}^{2}\right)$.

$C d=\frac{4}{3} \frac{\rho\left(\rho_{s 1}-\rho\right) g d p^{2}}{\mu^{2}} C_{d} R e^{2}=\frac{4}{3} \frac{\rho\left(\rho_{s 1}-\rho\right) g d_{p}^{3}}{\mu^{2}} C_{d} R e^{2}=\frac{4}{3} \frac{\rho\left(\rho_{s 1}-\rho\right) g d_{p}^{3}}{\mu^{2}}$

Em que $\rho$ é a densidade do fluido, $\rho s_{1}$ é a densidade do sólido, g é a gravidade, dp é o diâmetro da partícula e $\mu$ é a viscosidade do fluído.

A equação 4 representa o cálculo do número de Reynolds (Re).

$R e=\left[\left(\frac{K 1 C d R e^{2}}{24}\right)^{-1,2}+\left(\frac{C d R e^{2}}{K 2}\right)^{-0.6}\right]^{-\frac{1}{1,2}}$

A equação 5 é referente ao cálculo da velocidade terminal da partícula (vt), determinante para o cálculo do diâmetro de cada um dos elutriadores.

$v_{t}=\frac{\operatorname{Re} \mu}{d_{p} \rho}$

O cálculo do diâmetro dos três elutriadores pôde ser feito, por fim, por meio da equação 6

$D=\sqrt{\frac{4 Q}{d_{p} \rho}}$

Sendo que D é o diâmetro determinado do elutriador e Q é a vazão do fluído nos elutriadores, fixada em $1250 \mathrm{~cm}^{3} \mathrm{~s}-1$. Esses valores de diâmetros serão determinantes, pois serão utilizados na aplicação do modelo de elutriação.

Em posse dos diâmetros dos três elutriadores, foi possível avaliar a influência dos parâmetros alfa $(\alpha)$, velocidade mínima de fluidização (Umf) e vazão (Q) utilizados no modelo elaborado por Trabzuni, 2009.

A equação 7 mostra a taxa de alimentação das partículas no sistema.

$F_{0}(r i)=F_{0} P_{0}(r i)$ 
Em que F0(ri) é o fluxo da corrente da alimentação sólida por intervalo, F0 é o fluxo de alimentação de sólidos e P0(ri) é a fração em massa de cada intervalo de tamanho de partícula no fluxo de alimentação.

A equação 8 mostra o cálculo da massa de cada intervalo de partículas retido no leito.

$w\left(r_{i}\right)=\frac{F_{0}\left(r_{i}\right)-W\left(r_{i+1}\right) * \frac{\text { Rate }}{\Delta r_{i}}}{k\left(r_{i}\right)-\frac{\text { Rate }}{\Delta r_{i}}-3 * \frac{\text { Rate }}{r_{i}}}$

Dado que W(ri) é a massa de cada intervalo de partículas retido no leito, W(ri+1) é a massa de intervalo de partículas retida no ponto i+1 do leito, Rate é a taxa da reação e ri é o tamanho do intervalo ri. Na elutriação proposta para o atual sistema, foram desconsiderados os termos de reação química, uma vez que o objetivo principal foi apenas alcançar a separação das partículas.

O cálculo da massa da fração de cada intervalo de partículas no leito $(\mathrm{Pb}(\mathrm{ri}))$ é definido pela equação 9.

$P_{b}\left(r_{i}\right)=\frac{W\left(r_{i}\right)}{W}$

em que W é a massa total de sólidos no leito, definida pela equação 10.

$W=\sum W\left(r_{i}\right)$

A equação do fluxo de corrente de elutriação por intervalo (F2(ri)) é definida segundo a equação 11.

$F_{2}\left(r_{i}\right)=W\left(r_{i}\right) k\left(r_{i}\right)$

em que $\mathrm{k}($ ri) é definida como a constante da taxa de elutriação de sólidos.

A equação 12 representa o cálculo da fração em peso de cada partícula no intervalo de corrente de elutriação (P2(ri)).

$P_{2}\left(r_{i}\right)=\frac{F_{2}\left(r_{i}\right)}{F_{2}}$

em que F2 é o fluxo de elutriação de sóludos. A equação 13 representa o cálculo da constante da taxa de elutriação (k(ri)).

$k\left(r_{i}\right)=\rho_{s} v_{t}\left(1-\varepsilon_{i}\right)$

sendo zi o vazio da borda livre, cujo cálculo é definido pela equação 14 .

$\varepsilon_{i}=\left\{1+\frac{\lambda\left(U_{0}-U_{m f}\right)^{2}}{2 g D}\right\}^{-\alpha}$

em que U0 é a velocidade superficial do fluido utilizado, Umf é a velocidade mínima de fluidização e $\alpha$ é o expoente dependente do tamanho do intervalo de tamanho de partículas. Por fim, o cálculo de $\lambda$ é feito segundo a equação 15. 
$\frac{\lambda \rho_{s}}{d_{p}^{2}}\left(\frac{\mu}{\rho}\right)=12,3 R e^{-2,5} D$

Foi considerado um sistema de elutriação composto por três elutriados de diâmetros diferentes e pré-definidos, e uma solução com três partículas de densidades conhecidas, cada qual com distribuições granulométricas distintas. A velocidade mínima de fluidização (Umf), a vazão do sistema (Q) e o parâmetro alfa $(\alpha)$ foram variados individualmente cinco vezes, para que assim pudessem ser avaliados os comportamentos da massa das partículas retidas no leito (Wmf) e da constante de elutriação (k(ri)). A massa inicial para cada uma das porções de partícula foi considerada como $20 \mathrm{~g}$.

As simulações foram feitas utilizando as equações de 7 a 16, de modo a serem separadas em 3 casos: para o primeiro caso, foram mantidos a vazão Q e o parâmetro $\alpha$ constantes, alterando os valores de Umf; para o segundo caso, foram mantidos a vazão Q e Umf constantes, com variações sendo feitas nos valores de $\alpha$; para o terceiro caso, por fim, foram mantidos os parâmetros $\alpha$ e o fator multiplicativo da velocidade mínima de fluidização constantes, com variações em Q.

\section{Resultados e Discussão}

Os diâmetros encontrados para os elutriadores a partir das equações de 1 até 6 foram de 3,93 cm para o primeiro elutriador; $5,63 \mathrm{~cm}$ para o segundo elutriador e 7,43 $\mathrm{cm}$ para o terceiro elutriador.

\section{Caso 1}

Os resultados obtidos para análise da quantidade de massa retida no leito no estudo da variação da velocidade mínima de fluidização, mantendo Q e $\alpha$ constantes (correspondente às condições 1 a 5), são apresentados na Figura 2 a seguir.

Figura 2. Resultados para a variação de massa retida no leito quando variada a velocidade mínima de fluidização para (a) primeiro elutriador; (b) segundo elutriador; (c) terceiro elutriador.

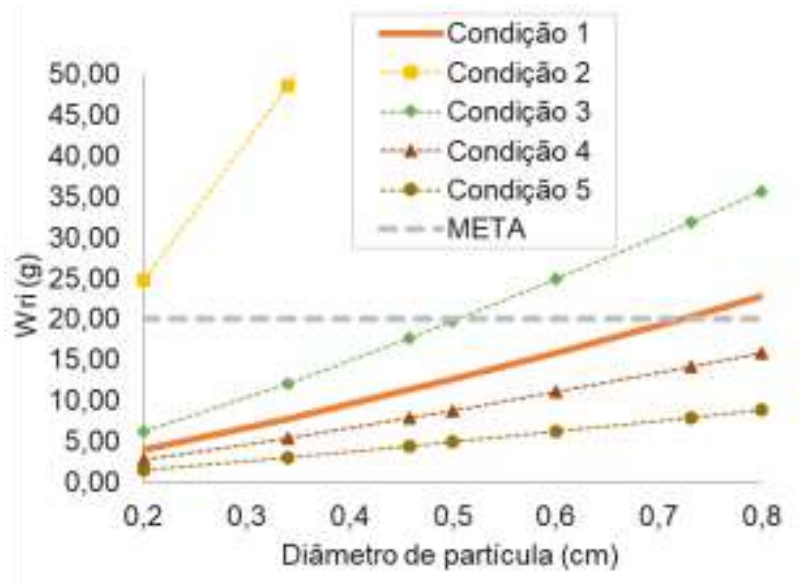

(a)

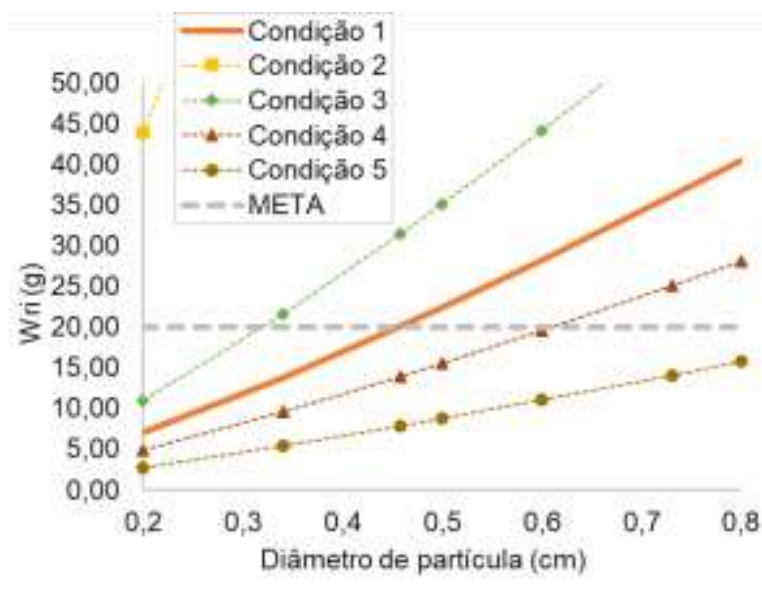

(b) 
Research, Society and Development, v. 10, n. 10, e537101019247, 2021

(CC BY 4.0) | ISSN 2525-3409 | DOI: http://dx.doi.org/10.33448/rsd-v10i10.19247

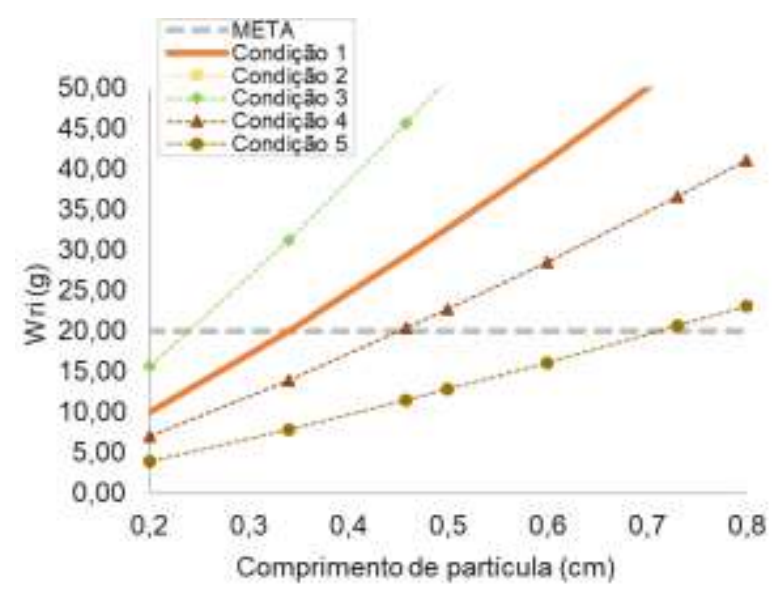

(c)

Fonte: Autores.

A Figura 3 mostra os resultados obtidos para análise da constante de elutriação no estudo da variação da velocidade mínima de fluidização $\left(\mathrm{U}_{\mathrm{mf}}\right)$, mantendo Q e $\alpha$ constantes.

Figura 3. Resultados para a variação de massa retida no leito quando variada a velocidade mínima de fluidização para (a) primeiro elutriador; (b) segundo elutriador; (c) terceiro elutriador.

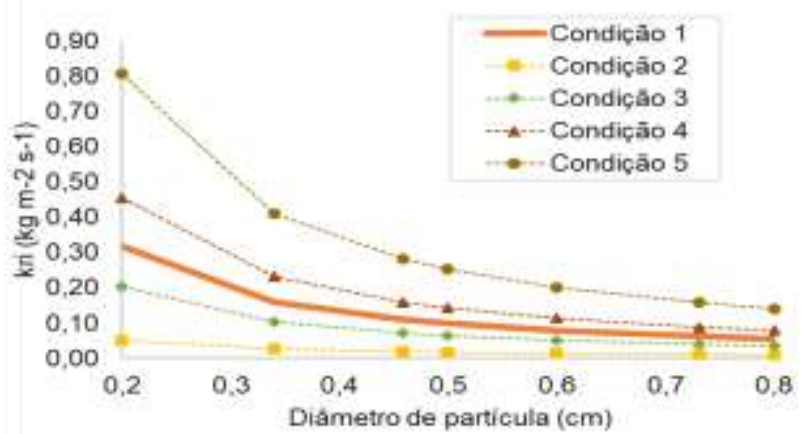

(a)

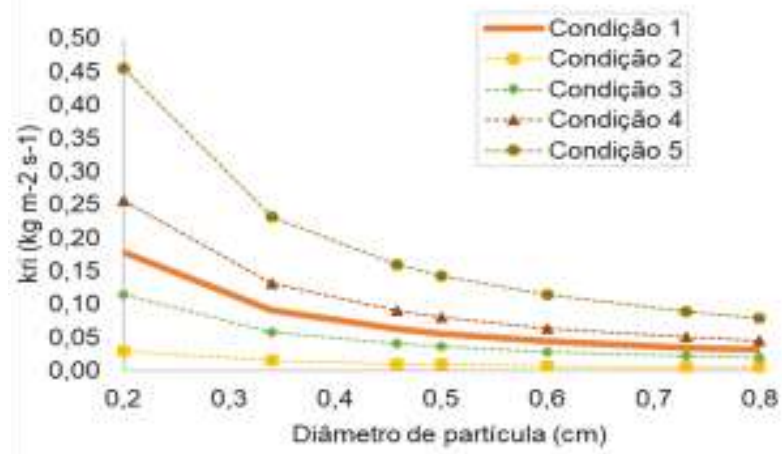

(b)

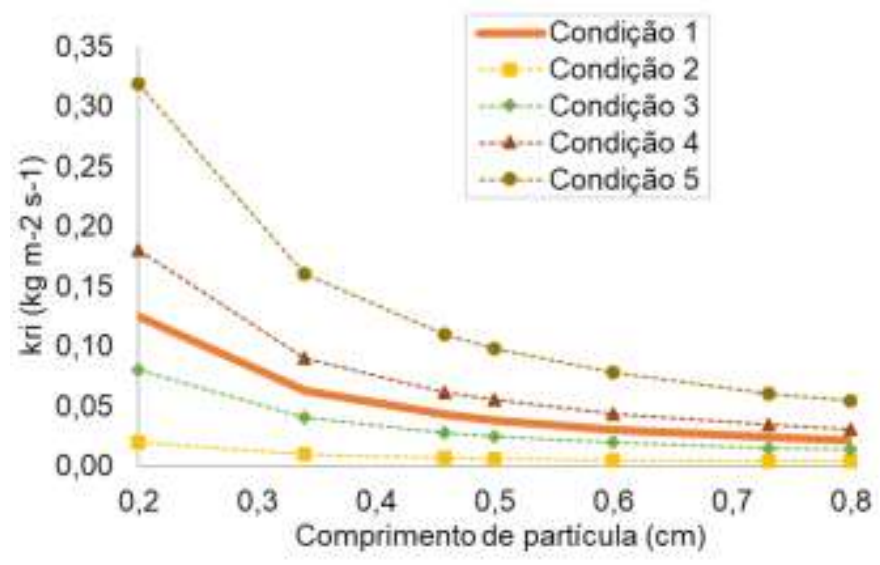

(c)

Fonte: Autores. 
Pode ser percebido que para o primeiro elutriador no tamanho de partícula de 0,7309 $\mathrm{cm}$ o valor ideal de $U_{\mathrm{mf}}$ para uma quantidade de massa de $20 \mathrm{~g}$ retidas no leito é de $51,52 \mathrm{~cm} \mathrm{~s}^{-1}$; para o segundo elutriador, no tamanho de partícula $0,4583 \mathrm{~cm}$, o valor ideal de $\mathrm{U}_{\mathrm{mf}}$ é de $25,11 \mathrm{~cm} \mathrm{~s}^{-1}$; para o terceiro elutriador, no tamanho de partícula $0,34 \mathrm{~cm}$, o valor ideal de $\mathrm{U}_{\mathrm{mf}}$ é de 14,41 $\mathrm{cm} \mathrm{s}^{-1}$, mostrando que condição 1 foi a condição ótima de operação para o caso 1 .

Além disso, é possível concluir que a diminuição no valor do fator da velocidade mínima de fluidização e, consequentemente, no valor de $\mathrm{U}_{\mathrm{mf}}$ implica em uma diminuição no valor de $\mathrm{W}\left(\mathrm{r}_{\mathrm{i}}\right)$ e em um aumento no valor de $\mathrm{k}\left(\mathrm{r}_{\mathrm{i}}\right)$. Ou seja, $\mathrm{W}\left(\mathrm{r}_{\mathrm{i}}\right)$ é diretamente proporcional a $\mathrm{U}_{\mathrm{mf}}$ e inversamente proporcional a $\mathrm{k}\left(\mathrm{r}_{\mathrm{i}}\right)$.

\section{Caso 2}

Os resultados obtidos para análise da quantidade de massa retida no leito no estudo da variação de $\alpha$, mantendo $Q$ e $U_{\mathrm{mf}}$ constantes (condições de 6 a 10), são apresentados na Figura 4 a seguir.

Figura 4. Resultados para a variação de massa retida no leito quando variado $\alpha$ (a) primeiro elutriador; (b) segundo elutriador; (c) terceiro elutriador.

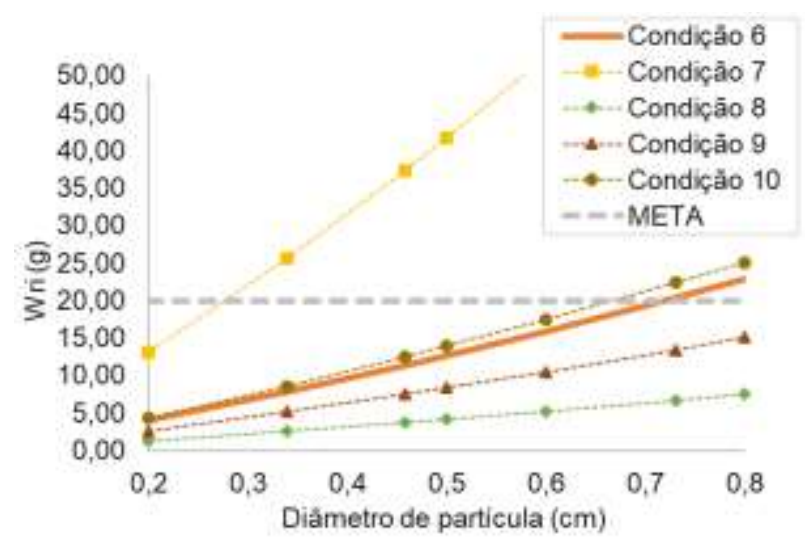

(a)

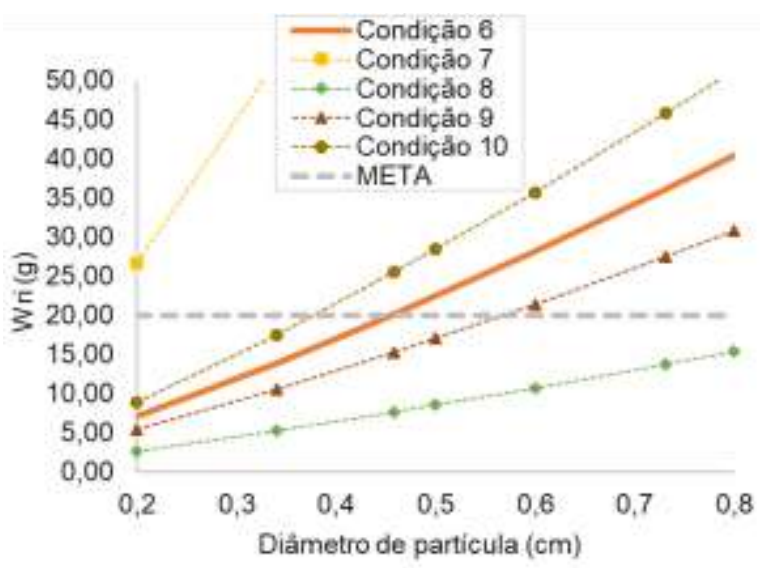

(b)

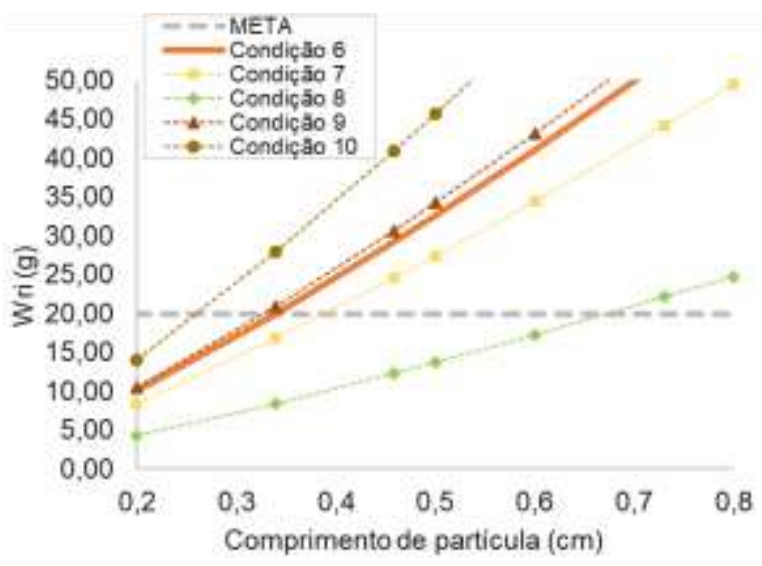

(c)

Fonte: Autores.

A Figura 5 mostra os resultados obtidos para análise da constante de elutriação no estudo da variação de $\alpha$, mantendo Q e $U_{\mathrm{mf}}$ constantes.

Pode ser percebido que para o primeiro elutriador, no tamanho de partícula de $0,7309 \mathrm{~cm}$, o valor ideal de $\alpha$ para 
uma quantidade de massa de $20 \mathrm{~g}$ retidas no leito é de 3,3; para o segundo elutriador, no tamanho de partícula $0,4583 \mathrm{~cm}$, o valor ideal de $\alpha$ é de 3,8; para o terceiro elutriador, no tamanho de partícula $0,34 \mathrm{~cm}$, o valor ideal de $\alpha$ é de 4,2 ; mostrando que a condição 6 foi a condição ótima de operação para o caso 2 .

Além disso, é possível concluir que o aumento do valor de $\alpha$ implica em uma diminuição no valor de $\mathrm{W}\left(\mathrm{r}_{\mathrm{i}}\right)$ e em um aumento no valor de $\mathrm{k}\left(\mathrm{r}_{\mathrm{i}}\right)$. Ou seja, $\mathrm{W}\left(\mathrm{r}_{\mathrm{i}}\right)$ é inversamente proporcional a $\alpha$ enquanto que $\mathrm{k}\left(\mathrm{r}_{\mathrm{i}}\right)$ é diretamente proporcional a $\alpha$.

Figura 5. Resultados para a variação de constante de elutriação quando variado $\alpha$ para (a) primeiro elutriador; (b) segundo elutriador; (c) terceiro elutriador.

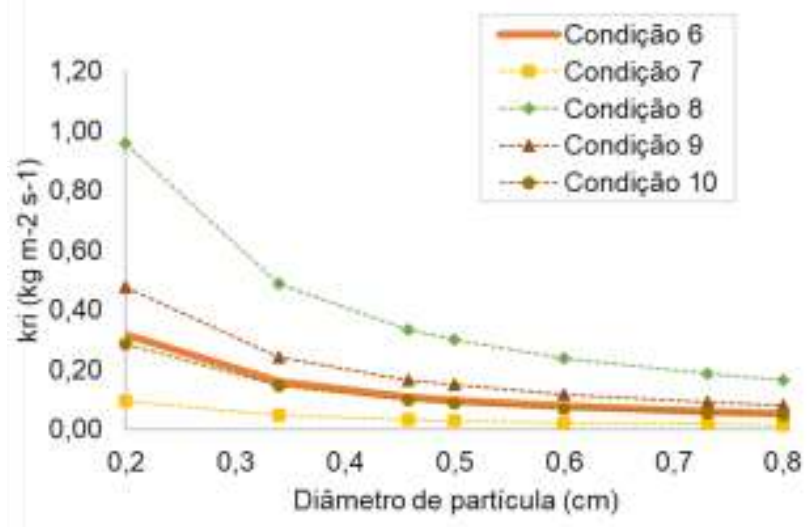

(a)

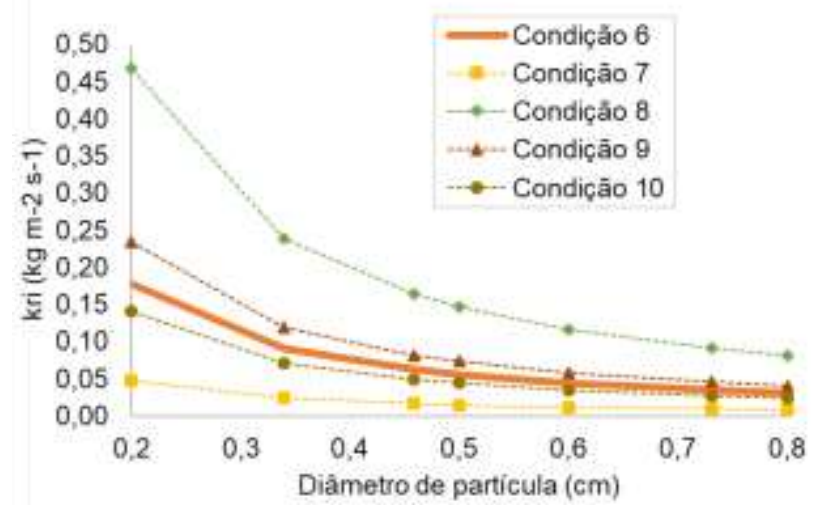

(b)

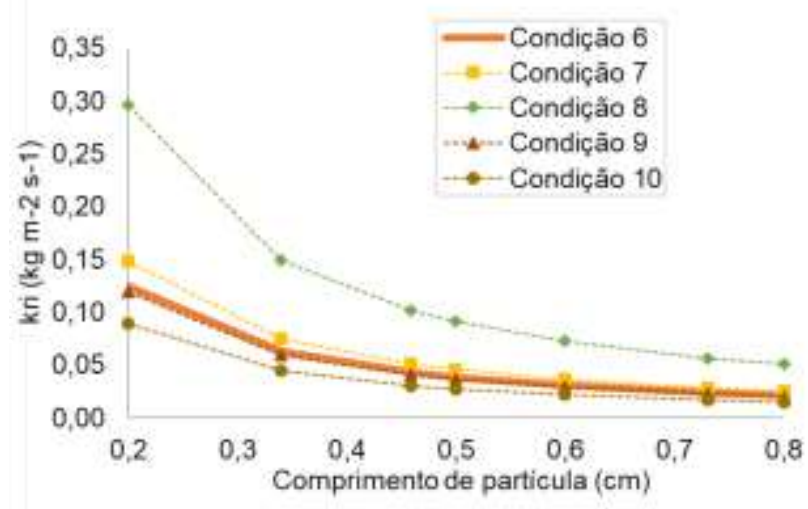

(c)

Fonte: Autores.

\section{Caso 3}

A Figura 6 representa os resultados obtidos para análise da quantidade de massa retida no leito no estudo da variação de Q, mantendo $\alpha$ constante (condições 11 a 15); $\mathrm{U}_{\mathrm{mf}}$ também sofrerá variação uma vez que o mesmo depende da vazão, no entanto o fator da velocidade mínima de fluidização é constante. 
Research, Society and Development, v. 10, n. 10, e537101019247, 2021

(CC BY 4.0) | ISSN 2525-3409 | DOI: http://dx.doi.org/10.33448/rsd-v10i10.19247

Figura 6. Resultados para a variação de massa retida no leito quando variado Q (a) primeiro elutriador; (b) segundo elutriador;

(c) terceiro elutriador.

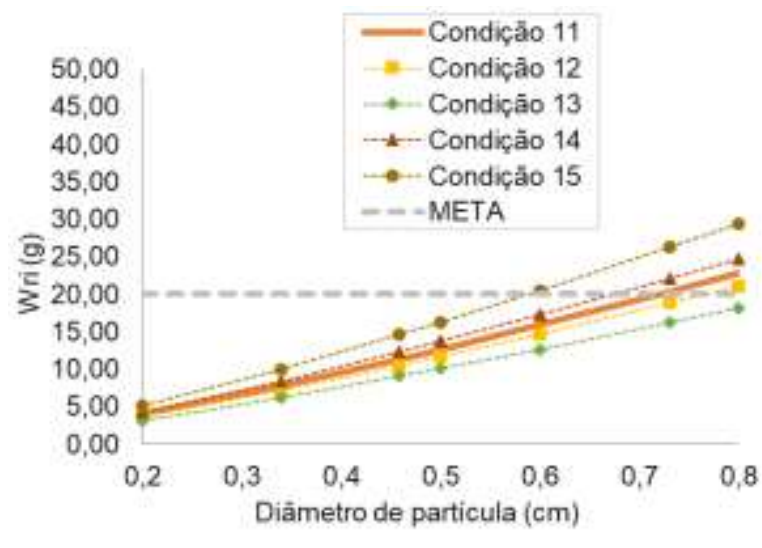

(a)

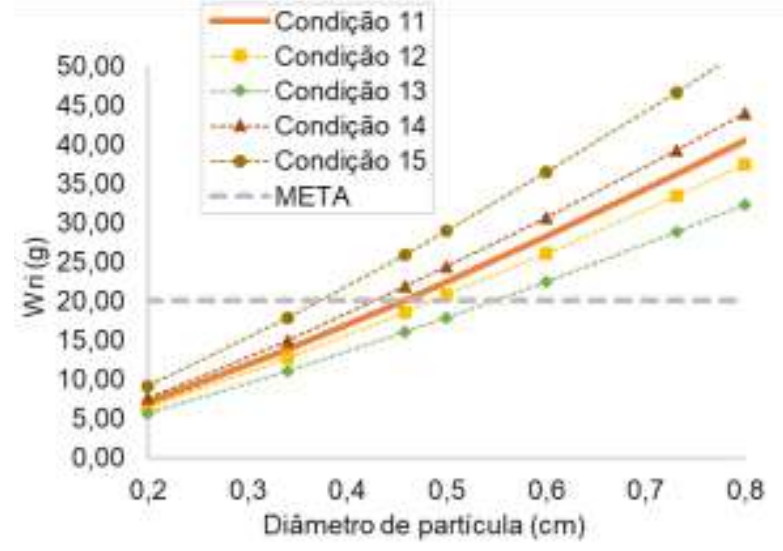

(b)

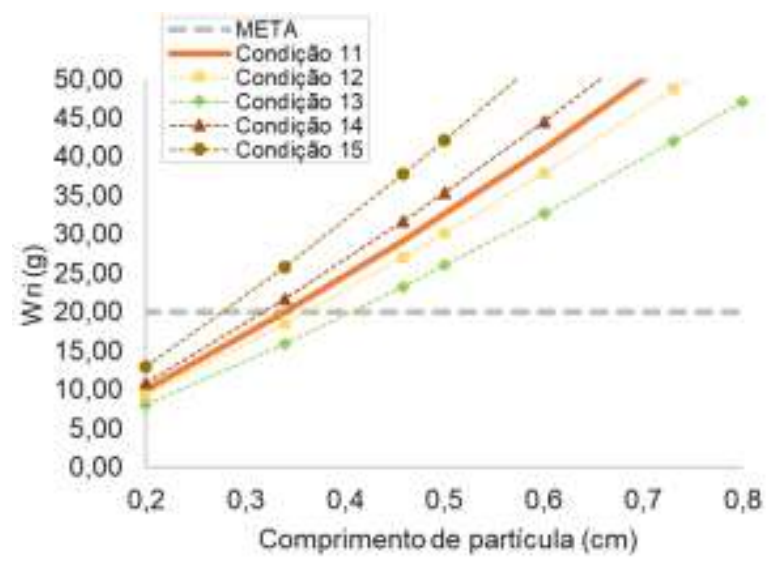

(c)

Fonte: Autores. 
A Figura 7 representa os resultados obtidos para análise da quantidade de massa retida no leito no estudo da variação de $\mathrm{Q}$, mantendo $\alpha$ e $\mathrm{U}_{\mathrm{mf}}$ constantes.

Figura 7. Resultados para a variação de constante de elutriação quando variado Q para (a) primeiro elutriador; (b) segundo elutriador; (c) terceiro elutriador.

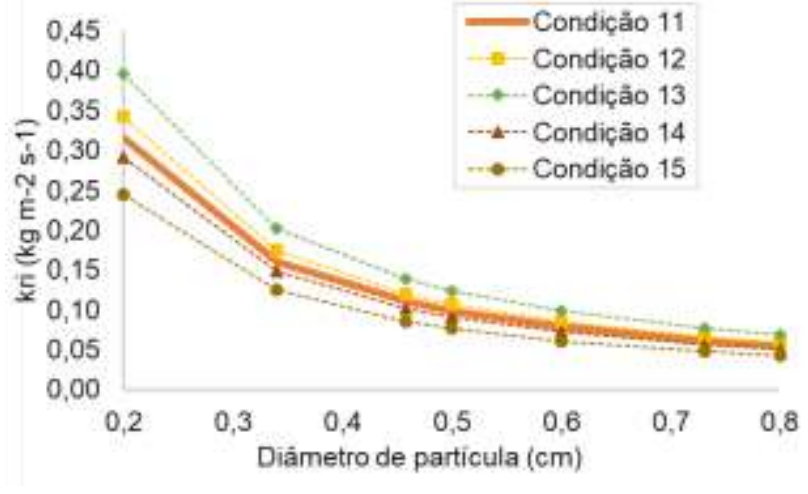

(a)

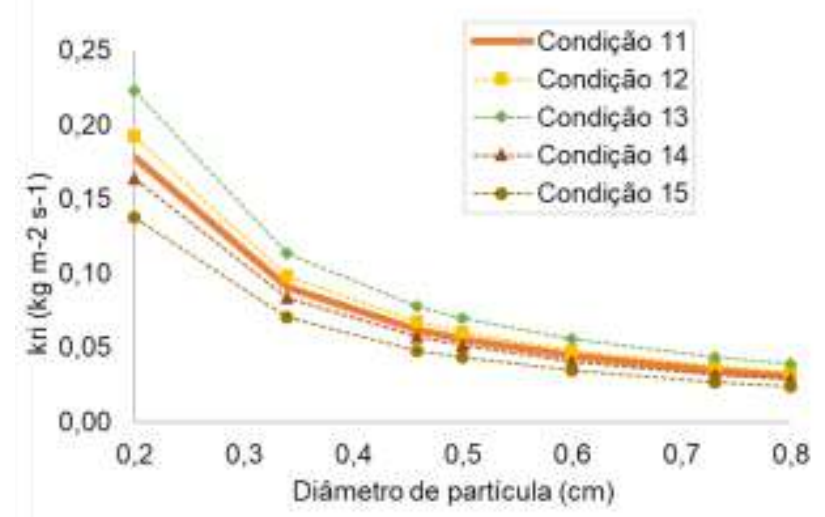

(b)

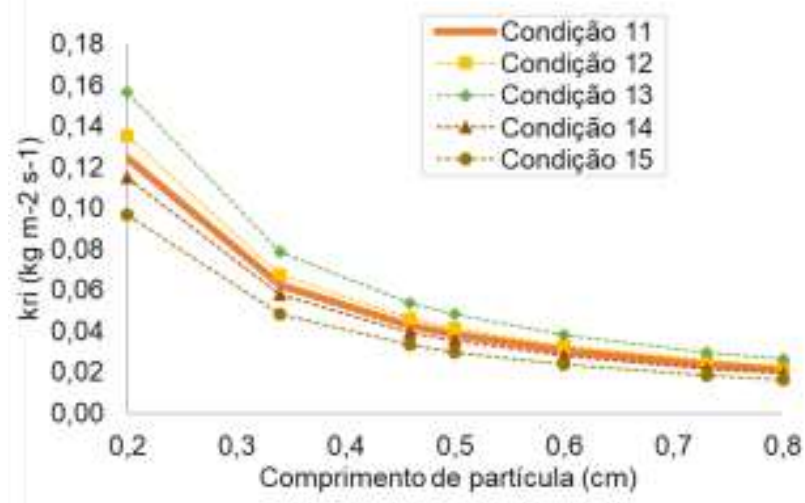

(c)

Fonte: Autores.

Pode ser percebido que o valor ideal de Q para uma quantidade de massa de $20 \mathrm{~g}$ retidas no leito é de $1250 \mathrm{~cm}^{3} \mathrm{~s}^{-1} \mathrm{para}$ todos os elutriadores, como mostra a condição 11 que foi a condição ótima de operação para o caso 3.

Além disso, é possível concluir que o aumento no valor de Q implica na diminuição do valor de $\mathrm{W}\left(\mathrm{r}_{\mathrm{i}}\right)$ e no aumento do valor de $\mathrm{k}\left(\mathrm{r}_{\mathrm{i}}\right)$. Ou seja, $\mathrm{W}\left(\mathrm{r}_{\mathrm{i}}\right)$ é inversamente proporcional a $\mathrm{Q}$ enquanto $\mathrm{k}\left(\mathrm{r}_{\mathrm{i}}\right)$ é diretamente proporcional a $\mathrm{Q}$.

É possível observar, a partir do Quadro 1, os parâmetros de Q, $\mathrm{U}_{\mathrm{mf}}$ e $\alpha$ os que possibilitaram as condições ótimas de operação para cada um dos operadores individualmente.

Quadro 1. Condições ótimas de operação para cada um dos elutriadores indivudualmente.

\begin{tabular}{|c|c|c|c|}
\hline Parâmetros & Elutriador 1 & Elutriador 2 & Elutriador 3 \\
\hline Vazão $\left(\mathrm{cm}^{3} \mathrm{~s}^{-1}\right)$ & 1250,00 & 1250,00 & 1250,00 \\
\hline$\alpha$ & 3,30 & 3,80 & 4,20 \\
\hline Umf $\left(\mathrm{m} \mathrm{s}^{-1}\right)$ & 51,52 & 25,11 & 14,41 \\
\hline
\end{tabular}

Fonte: Autores. 
Uma vez obtidas as condições ótimas de operação para os três elutriadores, foi feita a média dos valores de Q, fator da velocidade mínima de fluidização e de $\alpha$, de modo com que a operação pudesse ser aplicada aos três elutriadores em série. O Quadro 2 mostra as condições obtidas para os três elutriadores em série.

Quadro 2. Condições ótimas de operação para os elutriadores 1, 2 e 3 em série.

\begin{tabular}{|c|c|c|c|}
\hline Parâmetros & Elutriador 1 & Elutriador 2 & Elutriador 3 \\
\hline Wri $(\mathrm{g})$ & 17,84 & 20,26 & 22,25 \\
\hline $\mathrm{k}_{\mathrm{ri}}\left(\mathrm{g} \mathrm{m}^{-2} \mathrm{~s}^{-1}\right)$ & 0,07 & 0,06 & 0,06 \\
\hline $\mathrm{D}_{\text {elutriador }}(\mathrm{cm})$ & 3,93 & 5,63 & 7,43 \\
\hline$\rho_{\text {partícula }}\left(\mathrm{g} \mathrm{cm}^{-3}\right)$ & 7,40 & 5,50 & 2,42 \\
\hline Umf $\left(\mathrm{m} \mathrm{s}^{-1}\right)$ & 51,51 & 25,11 & 14,41 \\
\hline
\end{tabular}

Fonte: Autores.

Foi possível observar que os valores de $\mathrm{W}\left(\mathrm{r}_{\mathrm{i}}\right)$ para a aplicação dos três elutriadores em série não manteve-se tão próxima de $20 \mathrm{~g}$ (condição ideal) tanto quanto proposto para os elutriadores separados. Isso ocorreu, pois em posse dos valores, foi necessária a utilização da média de $\alpha$, o que ocasionou ligeiras mudanças nos valores anteriormente propostos de $\mathrm{W}\left(\mathrm{r}_{\mathrm{i}}\right)$ e $\mathrm{k}\left(\mathrm{r}_{\mathrm{i}}\right)$.

Embora os valores tenham diferido dos valores propostos para os elutriadores separados, é possível concluir que houve a elutriação das partículas de propostas quando os elutriadores operaram em série, evidenciando uma elutriação bem sucedida.

\section{Considerações Finais}

Após aplicar as diferentes condições nas variáveis de vazão (Q), velocidade mínima de fluidização (Umf) e parâmetro alfa $(\alpha)$, foi possível perceber que significativas alterações na vazão do fluido não resultaram em variações expressivas na quantidade de massa de partículas retida no leito (W(ri)) e na constante de elutriação (k(ri)),diferentemente de variações no parâmetro alfa $(\alpha)$ e na velocidade mínima de fluidização (Umf), em que pequenas flutuações nos valores simulados alteravam significativamente as variáveis analisadas.

Para cada um dos elutriadores, analisados individualmente com determinada partícula em uma faixa de tamanho préestabelecida, foram encontradas diferentes condições ótimas para os parâmetros alfa $(\alpha)$ e velocidade mínima de fluidização (Umf), enquanto os valores ideais de vazão (Q) encontrados foram os mesmos para os três elutriadores. Para análise dos três elutriadores operando em série, foi feita, portanto, uma média entre as condições ótimas dos parâmetros citados.

As simulações, adaptadas do trabalho de Trabzuni, 2009, foram satisfatórias para o caso estudado, e a elutriação proposta levando em considerações as características físicas das partículas 1, 2 e 3 é bem-sucedida quando os elutriadores operam em série, evidenciando resultados coerentes com o que era esperado a partir da simulação feita no presente trabalho.

Os autores deixam como sugestões para trabalhos futuros a realização de testes com as dimensões, vazão e condições estabelecidas como ótimas neste trabalho.

\section{Referências}

Amaral, A. J. R., \& Filho, C. A. L. (2021). Mineração. https://www.dnpm-pe.gov.br/Geologia/Mineracao.php

Colakyan, M., \& Catipovic, N., \& Jovanovic, G. \& Fitzgerald, T. (1979). Elutriation from a Large Particle Fluidized Bed with and without Immersed Heat Transfer Tubes. AIChE Symposium Series, 77 (205), 66-75. https://pascal-francis.inist.fr/vibad/index.php?action=getRecordDetail\&idt=PASCAL8130426292

Cremasco, M. A. (2014). Operações Unitárias em Sistemas Particulados e Fluidodinâmicos. Blücher.

Cremasco, M. A. (2014). Vale a pena estudar Engenharia Química. Blücher.

Englert, A.H., \& Rubio, J. (2011) Beneficiamento de rejeito piritoso do processamento de carvão mineral por elutriação aquosa. 
Research, Society and Development, v. 10, n. 10, e537101019247, 2021

(CC BY 4.0) | ISSN 2525-3409 | DOI: http://dx.doi.org/10.33448/rsd-v10i10.19247

Follmer, L. R., \& Beavers, A. H. (1973). An Elutriator Method for Particle-size Analysis with Quantitative Silt Fractionation. Journal of Sedimentary Research, 43(2), 544-549.

\section{$10.1306 / 74 d 727 d e-2 b 21-11 d 7-8648000102 c 1856 d$}

Foust, A.S., \& Wenzel, L. A., \& Clump, C.W., \& Maus, L., \& Andersen, L.B. (1982). Princípio das Operações Unitárias. Guanabara Dois.

Geankoplis, C.J. (2006). Procesos de transporte y principios de procesos de separación. Compañía Editorial Continental.

Gomide, R. (1980). Operações Unitárias: separações mecânicas. Câmara Brasileira do Livro (Edição do autor)

Guha, S. K., \& Kumar, A., \& Gupta, P. S (1972). Mechanism of Elutriation fromFluidized Beds. The Canadian Journal of Chemical Engineering, 50(5), 602606. $10.1002 /$ cjce. 5450500508

Hettler, E. N., \& Gulliver, J. S., \& Kayhanian, M. (2011). An elutriation device to measure particle settling velocity in urban runoff. Science of the Total Environment, 409(24), 5444-5453. 10.1016/j.scitotenv.2011.08.045

Choi, J. H., \& Sun, J. M., \& Chang, I. Y. \& Shun, D.W., \& Yi, C. K., \& Son, J. E., \& Kim, S. D. (2001). The effect of fine particles on elutriation of coarse particles in a gas fluidized bed. Powder Technology, 121 (2-3), 190-194. 10.1016/s0032-5910(01)00333-3

Klinger, C.; \& Leidens, N.; \& Nunes, I. S. (2017). Construção de módulo de elutriação como ferramenta de contextualização para o ensino de operações unitárias. IV Congresso Internacional de Educação Científica e Tecnológica, 10. (anais)

Kloeckner, J.; \& Englert, A. H.; \& Rubio, J. (2011). Recuperação do mineral pirita (FeS2) de rejeitos do processamento de carvão por elutriação aquosa.. XX Feira de Iniciação à Inovação e ao Desenvolvimento Tecnológico, p.3. (anais)

Massarani, G. (2001). Fluidodinâmica em sistemas particulados. E-Papers.

Merrick, D., \& Highley, J. (1974). Particle Size Reduction and Elutriation in a Fluidized Bed Process. AIChE symposium series. 70(137), 366-378.

Perry, R.H., \& Chilton, C.H. (1986). Manual de Engenharia Química. Guanabara Dois.

Silva, E. R. (2016). Análise experimental e numérica do sistema de separação via elutriação de sedimento de drenagem.

Trabzuni, F. (2009). Simulation of titanium dioxide (TiO2) ore elutriation from chlorination fluidized bed reactors. The Journal of The Southern African Institute of Mining and Metallurgy, 109(2), 129-133. http://www.scielo.org.za/scielo.php?script=sci_arttext\&pid=S2225-62532009000200006

Wamburg, J. (2014). Estudo confirma impactos da mineração no país. Agência Brasil. https://agenciabrasil.ebc.com.br/pesquisa-e-inovacao/noticia/201412/estudo-do-cetem-confirma-impactos-da-mineracao-no-pais

Wen, C. Y., \& Chen, L. H. (1982). Fluidized bed freeboard phenomenona: Entrainment and elutriation. AIChE Journal, 28(1), 117-128. 10.1002/aic.690280117

Yates, J. G. (1983). Fundamentals of Fluidized Bed Chemical Processes: Butterworth. 